\title{
Evaluation of the nursing process and use of electronic documentation in an acute hospital in Switzerland
}

\author{
Michelle Bruylands, Wolter Paans, Hannele Hediger, Maria Müller Staub
}

Zürcher Hochschule angewandter Wissenschaft (ZHAW), Departement Gesundheit

\section{Research questions}

The main purpose of this study was to evaluate the impact of the educational programme "Guided Clinical Reasoning" (GCR) on the quality of nursing documentation in an acute hospital in Switzerland. Additionally the effect of the introduction of an intelligent electronic nursing documentation expert system (e-doc) and simultaneous cessation of GCRwere examined in 2011.

\section{Methods}

To evaluate the impact of GCR, a cross-sectional evaluation study was conducted at three measurement points in the years 2005, 2006 and 2011. Each sample of 36 documents was rated by the instrument "Quality of Nursing Diagnoses, Interventions and Outcomes" (Q-DIO) and the results were compared with a quantitative design. To study the effects of the e-doc, a descriptive design was used. The amount of chosen nursing diagnoses and their degree of content accuracy was analysed for both nurses and the electronic documentation system. Furthermore the level of correct "decision support" in hypothetical nursing diagnosis by the e-doc and the use of this offer by nurses were compared.

\section{Findings}

The GCR programme showed the best effects regarding the quality of the nursing process in 2006. Despite implementing an intelligent e-doc system as a decision support tool, measurement time point three (2011) provided the lowest scores, except for phrasing accurate nursing diagnoses. The quality of documentation regressed to the level of 2005, one year after implementing nursing diagnostics. The skills for using the NNN (NANDA, NIC, NOC) nursing process accurately, acquired in 2006, did not sustain. While nurses wrote detailed reports, they used a limited amount of recurring diagnoses. In comparison e-doc consistently and abundantly provided correct hypothetical nursing diagnoses. Despite this support, they were scantly used by nurses.

\section{Conclusions}

Being at close temporal proximity the first two measurement points provided constant training for nurses in GRC and a high documentation quality was achieved. To accurately depict the nursing process, an electronic support system alone did not suffice, particularly as the GCR programme was terminated. Electronic support systems can assist in conducting a theory-based nursing process, but clinical reasoning is essential for meaningful use of e-docs. Constant support of nurses and awareness of barriers in conducting the nursing process and using intelligent decision supporting tools are crucial for a high level of quality in nursing documentation.

\section{Correspondence:}

Zürcher Hochschule angewandter Wissenschaft (ZHAW) Departement Gesundheit

Technikumstrasse 71

Postfach

$\mathrm{CH}-8401$ Winterthur

bruy[at]zhaw.ch 